\title{
Refinement and Verification of Synchronized Component-based Systems
}

\author{
Olga Kouchnarenko - Arnaud Lanoix
}

$\mathbf{N}^{\circ} 4862$

Juin 2003

THÈME 2 



\title{
Refinement and Verification of Synchronized Component-based Systems
}

\author{
Olga Kouchnarenko* , Arnaud Lanoix ${ }^{\dagger}$ \\ Thème 2 - Génie logiciel \\ et calcul symbolique \\ Projet CASSIS \\ Rapport de recherche $\mathrm{n}^{\circ} 4862$ - Juin 2003 - 29 pages
}

\begin{abstract}
This article deals with specification, refinement and verification approaches for systems designed with synchronized components. First of all, we define a synchronized composition of components. Transition systems are used to specify or/and to model synchronized component-based systems. Second, we give refinement semantics for these component-based systems before proposing a method to verify the refinement of a whole system from the weak refinement of its components. We also present SynCo (for Synchronized Component-based Systems): a tool we are implementing using our method. Third, a compositional way to verify safety properties is proposed: the unreachability of a (set of) state(s) can be efficiently ensured for a synchronized component-based system.

The different aspects of our work are illustrated on an industrial example of a wind-screen wipers system composed of a control lever, a rain sensor and two (left and right) wind-screen wipers.
\end{abstract}

Key-words: composition, synchronization, compositional verification, refinement, reachability analysis

* LIFC - Université de Franche-Comté, email: kouchna@univ-fcomte.fr

† LIFC - Université de Franche-Comté, email: lanoix@univ-fcomte.fr 


\section{Raffinement et vérification de systèmes à composants synchronisés}

Résumé : Cet article traite de la spécification, du raffinement et de la vérification de systèmes conçus sous forme de composants synchronisés. Pour spécifier et/ou pour modéliser ces systèmes, nous utilisons des systèmes de transitions. Nous définissons d'abord une composition synchronisée de systèmes et donnons la sémantique du raffinement pour ce type de systèmes. Ensuite nous donnons des conditions nécessaires et suffisantes du raffinement du système complet à partir du raffinement affaibli de ses composants. Un algorithme de vérification du raffinement pour ce type de système est implanté dans SynCo (pour Synchronized Component-based Systems) Finalement, un algorithme de vérification compositionnelle de propriétés de sûreté est proposé.

Les différents aspects de ce travail sont illustrés par l'exemple industriel d'un controleur d'essuyage composé d'un levier de controle, d'un capteur de pluie et de deux (gauche et droite) essuie-glaces.

Mots-clés : composition, synchronisation, vérification compositionnelle, raffinement, vérification algorithmique, analyse d'atteignabilité 


\section{Introduction}

Verification across abstraction and refinement steps is a central and important issue in formal system validation. It presents both practical and theoretical difficulties that have not yet been satisfactory solved.

In this paper, we deal with specification and verification of component-based finite state systems supporting a top-down refinement paradigm. We suppose a specification obtained by a refinement process. Several methods, like $B[1,4], T L A+[20]$, $C S P 2 B$ [12], etc. propose a refinement based development. The system specification and modelling we consider in this paper, are inspired by the syntactic and semantic concepts of the $B$ refinement method which has been successfully used to specify many reactive systems. On overview, the reader can refer to case studies such as an industrial automatism [2], as well as industrial applications such as MÉTÉOR [5] by Matra Transport International, and the SPECTRUM project [28] by GEC-Marconi Avionics Limited.

In $[16,18]$, it has been proposed to enrich $B$ specifications with dynamic properties formulated in the Propositional Linear Temporal Logic $(P L T L)$. In [8], we express the refinement semantics as a relation between transition systems. In general, behavioural properties established for an abstract system model are not preserved when the system is refined to a richer level of detail. It is not the case for us: in [14], we show that our refinement relation preserves the abstract system $P L T L$ properties. This way, an algorithmic verification of the refinement by model exploration can be associated with the verification of the PLTL properties, by model-checking.

However, it is well-known that the algorithmic verification quickly meets its limits when applied to huge systems. Therefore, we have to face the problem of combinatorial explosion during refinement since details introduced by the refinement tend to drastically increase the number of states of the systems. It is also the case while verifying properties by model-checking. Compositional approaches partially avoid this problem.

A way to have components in the $B$ method is to decompose the application into separate machines. There are many works on structured development using decomposition into machines and refinement (see, for example [13, 11, 27]). Generaly, $B$ event systems which are closed systems can be used to describe the components. So, the interactions between components have to be described independently. This is the idea used for example in [12] or in [29].

Unlike the Schneider and Treharne's approach [26], we propose in [9] to remain in the framework of the $B$ event systems. We assume that components do not share variables and we propose to specify a synchronization as pairs of events belonging

$\mathrm{RR} \mathrm{n}^{\circ} 4862$ 
to two different components with feasibility conditions. These conditions achieve the synchronization by constraining the activation of events of a component by a predicate over the variables of another component. Moreover, during the refinement verification we take the external non-determinism into account.

In this paper, we go further to conciliate the synchronized component-based specification with the refinement verification. First, we propose to use transition systems both for specifying and for modelling synchronized component-based systems. Second, we define a refinement relation of component-based systems which is weaker than the refinement relation defined in [9]. Third, we give the conditions to ensure the refinement of the whole system from the weak refinements of its synchronized components, and vice versa. Furthermore, we propose to exploit the refinement to verify safety properties in a compositional way. The interest of this proposition is that the component-based refinement guarantees preservation of the abstract systems properties for the refined systems.

As a comparison, our goal for addressing the $B$ event systems composition is different from the $B$ decomposition one proposed by J.-R. Abrial. In [3], the event systems decomposition is based on extern shared variables and synchronized events. The main advantage of our technique is that it frees the user from the whole system design.

This paper is organised as follows. After giving preliminary notions, we define, in Section 2, the behavioural semantics of synchronized component-based systems. In Section 3, we define a refinement relation for these systems and explain our main compositionality result using for the compositional refinement verification. Then, in Section 4, we investigate how our approach is used in the context of a compositional safety property verification. Section 5 introduces a tool implementing the synchronized component refinement verification. Throughout this paper, we illustrate the use of our framework on an industrial example of a wind-screen wipers system. We end by some perspectives.

\section{Synchronized Parallel Composition}

In this section, we introduce interpreted labelled transition systems to specify and to model the behaviours of a component. We specify the synchronized behaviours of components by tuples of labels with feasibility conditions constraining activations of transitions with these labels. We provide a definition for a context-in component (i.e. a component in the context of the others). This definition is required to define the synchronized parallel composition of components under a synchronization. 
Let $\operatorname{Var}=\left\{X_{1}, \ldots, X_{n}\right\}$ be a finite set of variables with their respective domains $\mathbb{D}_{1}, \ldots, \mathbb{D}_{n}$. Let $A P$ be a set of atomic propositions $a p \stackrel{\text { def }}{=}\left(X_{i}=v\right)$ with $X_{i} \in V a r$ and $v \in \mathbb{D}_{i}$. Let $S P$ be a set of state propositions $s p$ defined by following grammar: $s p_{1}, s p_{2}::=a p\left|\neg s p_{1}\right| s p_{1} \vee s p_{2}$.

Definition 1 (Interpreted Labelled Transition System (LTS))

$A$ interpreted labelled transition system $S$ over $\operatorname{Var}$ is a tuple $<Q, Q_{0}, E, T, l>$ where:

- $Q$ is a set of states,

- $Q_{0} \subseteq Q$ is a set of initial states,

- $E$ is a finite set of transitions labels or actions,

- $T \subseteq Q \times E \times Q$ is a labelled transition relation, and

$-l: Q \rightarrow S P$ is an interpretation of each state on the system variables.

We define the sum of two transition systems over the same set of variables $V a r$. Furthermore, we will show how to compute a parallel composition of synchronized components using this operator.

\section{Definition 2 (Sum of Two LTSs)}

Let $S_{1}=<Q_{1}, Q_{01}, E_{1}, T_{1}, l_{1}>$ and $S_{2}=<Q_{2}, Q_{02}, E_{2}, T_{2}, l_{2}>$ be two transition systems over Var. The sum of $S_{1}$ and $S_{2}$, written $S_{1} \uplus S_{2}$, is $<Q_{1} \cup Q_{2}, Q_{01} \cup$ $Q_{02}, E_{1} \cup E_{2}, T_{1} \cup T_{2}, l_{12}>$ where $l_{12}$ is defined by:

$$
l_{12}(q)=\left\{\begin{array}{l}
l_{1}(q) \text { if } q \in Q_{1} \\
l_{2}(q) \text { if } q \in Q_{2}
\end{array}\right.
$$

Moreover, $\forall q_{1} \cdot q_{1} \in Q_{1}, \forall q_{2} \cdot q_{2} \in Q_{2} .\left(l_{1}\left(q_{1}\right)=l_{2}\left(q_{2}\right) \Leftrightarrow q_{1}=q_{2}\right)$.

In order to define our synchronized parallel composition, we give the definition of a synchronization of $n$ components. For that, we introduced a new transition label '-' for the fictive action "skip".

\section{Definition 3 (Synchronization of $n$ Components)}

Let $S_{1}, \ldots, S_{n}$ be $n$ components. A synchronization Synch is a set of elements $(\alpha$ when $p$ ) where:

- $\alpha=\left(e_{1}, \ldots, e_{n}\right) \in \prod_{i=1}^{n}\left(E_{i} \cup\{-\}\right)$,

- $p$ is a state proposition on the components variables.

To illustrate the previous definitions, we introduce the example of a car windscreen wipers system. Consider the wipers system $W S_{A}$ composed by a control lever,

$\mathrm{RR} \mathrm{n}^{\circ} 4862$ 
a rain sensor and two (left and right) wind-screen wipers. The control lever $C O_{A}$ can select the mode of the wiper system: $c o=$ manual, $c o=$ auto or $c o=$ stop. Its behaviour is shown in Fig. 1. The left and the right wipers have the same behaviour. The transition system in Fig. 2 shows two positions for the left wiper $L W_{A}$ : lw=left $U p$ or $l w=l e f t D o w n$. It is the same thing for the right wiper $R W_{A}$. The rain sensor $S E_{A}$ can detect the rain amount ( $s e=n o t, s e=t i n y$ or $s e=s t r o n g$ ). Moreover, it can be off $(s e=o f f)$ (see Fig. 3).

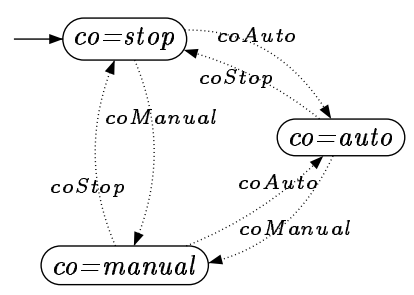

Figure 1: $C O_{A}$

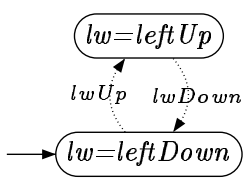

Figure 2: $L W_{A}$

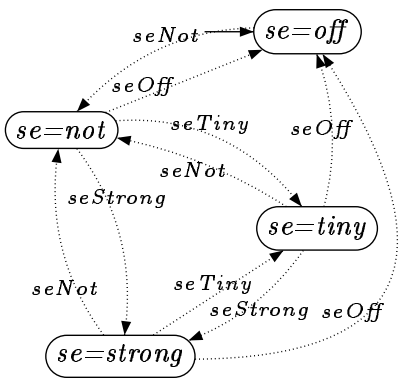

Figure 3: $S E_{A}$

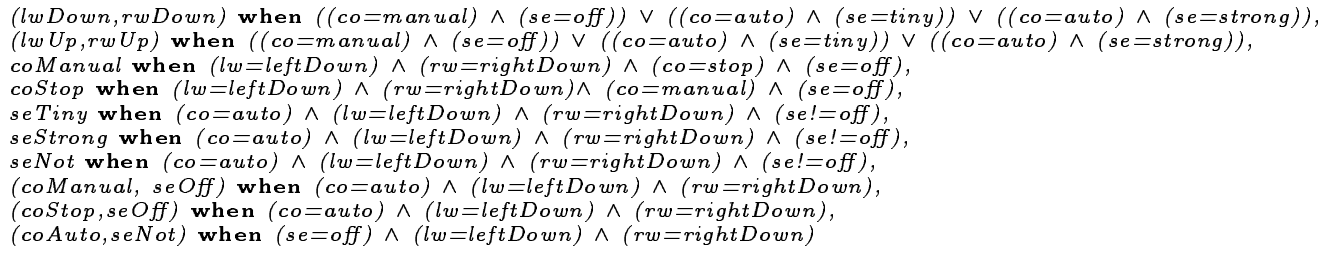

Figure 4: Synchronization $c s w_{A}$

A synchronization $\operatorname{csw}_{A}$ is given to describe the authorized behaviours of the complete system $W S_{A}$. For example, the left and right wipers must move together $((l w D o w n, r w D o w n)$ and $(l w U p, r w U p))$, the rain sensor sends information only if the control lever mode is auto (seTiny, (seStrong and seNot), the control lever mode or the rain sensor can change only if the wipers are down ((coManual,seOff), (coAuto,seNot)), etc. $c s w_{A}$ is given in Fig. 4. For readability reason the fictive transition label '-' is not shown in $c s w_{A}$.

Each component $S_{1}, \ldots, S_{n}$ is a context-free component. However, to take synchronization into account, we need to define a context-in component, i.e. a component in the context of the others under a synchronization Synch. 


\section{Definition 4 (Context-in Component)}

Let $S_{1}, \ldots, S_{n}$ be $n$ components. Let Synch be their synchronization. A context-in component $S_{i}^{c}$ is defined by the tuple $<Q_{i}^{c}, Q_{0 i}^{c}, E_{i}^{c}, T_{i}^{c}, l_{i}^{c}>$ where:

- $Q_{i}^{c} \subseteq Q_{1} \times \ldots \times Q_{n}$ with $\left(q_{1}, \ldots, q_{n}\right) \in Q_{i}^{c}$,

- $Q_{0 i}^{c} \subseteq Q_{01} \times \ldots \times Q_{0 n}$,

- $E_{i}^{c}=\left\{\left(e_{1}, \ldots, e_{i}, \ldots, e_{n}\right) \mid\left(\left(\left(e_{1}, \ldots, e_{i}, \ldots, e_{n}\right)\right.\right.\right.$ when $\left.p\right) \in$ Synch $)$

$\left.\wedge\left(e_{i} \in E_{i}\right)\right\}$

- $l_{i}^{c}\left(\left(q_{1}, \ldots, q_{n}\right)\right)=l_{1}\left(q_{1}\right) \wedge \ldots \wedge l_{n}\left(q_{n}\right)$,

- $T_{i}^{c} \subseteq Q_{i}^{c} \times E_{i}^{c} \times Q_{i}^{c}$ with

$\left(\left(q_{1}, \ldots, q_{n}\right),\left(e_{1}, \ldots, e_{n}\right),\left(q_{1}^{\prime}, \ldots, q_{n}^{\prime}\right)\right) \in T_{i}^{c}$ iff:

- $\left(\left(e_{1}, \ldots, e_{n}\right)\right.$ when $\left.p\right) \in$ Synch,

- $l_{i}^{c}\left(\left(q_{1}, \ldots, q_{n}\right)\right) \Rightarrow p$, and

$-\forall k .\left(k \in\{1, \ldots, n\} \Rightarrow\left(\left(e_{k}=-\wedge q_{k}=q_{k}^{\prime}\right) \vee\left(e_{k} \neq-\wedge\left(q_{k}, e_{k}, q_{k}^{\prime}\right) \in\right.\right.\right.$

$\left.\left.T_{k}\right)\right)$ ).

All context-in components have the same set of variables $\operatorname{Var}=\bigcup_{j=1}^{n} \operatorname{Var}_{j}$. Note that the graph representing a context-in component may be unconnected.

Figure 5 shows a representation of the context-in component $S E_{A}^{c}$ that presents all behaviours of $S E_{A}$ under $c s w_{A}$. It is either simple behaviours (seNot, seTiny, seStrong) or synchonized behaviours ((seOff,coManual), (seNot,coAuto)). We have similar figures for the context-in components $C O_{A}^{c}, L W_{A}^{c}$ and $R W_{A}^{c}$. Remark

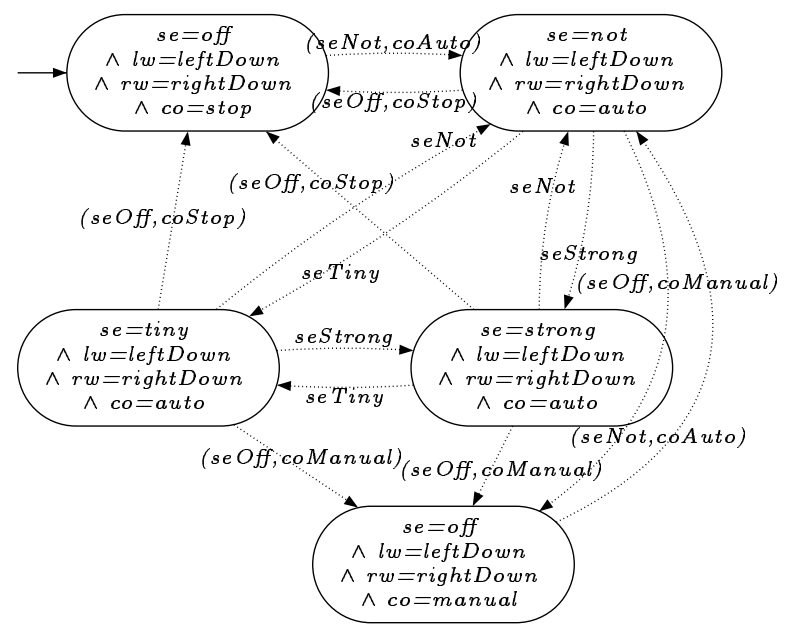

Figure 5: $S E_{A}^{c}$

$\mathrm{RR} \mathrm{n}^{\circ} 4862$ 
that $R W_{A}^{c}$ and $L W_{A}^{c}$ are identical. Indeed, all behaviours of $L W_{A}$ and $R W_{A}$ are synchronized ((lwDown,rwDown) and (lwUp,rwUp)).

The whole system is a rearrangement of its separate parts, i.e., the components and their interactions. This arrangement is specified by a synchronized composition between components. We define a synchronized composition of $n$ components $S_{1}, \ldots, S_{n}$ under a synchronization $S y n c h$ by the sum of the $n$ context-in components $S_{1}^{c}, \ldots, S_{n}^{c}$.

\section{Definition 5 (Synchronized Composition of $n$ Components)}

Let $S_{1}, \ldots, S_{n}$ be $n$ components and Synch their synchronization. Let $S_{1}^{c}, \ldots, S_{n}^{c}$ be their respective context-in components. The synchronized parallel composition of $S_{1}, \ldots, S_{n}$ under Synch is defined by:

$$
\|_{\text {Synch }}\left(S_{1}, \ldots, S_{n}\right) \stackrel{\text { def }}{=} \biguplus_{i=1}^{n}\left(S_{i}^{c}\right)
$$

Notice that our parallel composition is more expressive than the classical synchronized product of transition systems. This is due to feasibility conditions, i.e., predicates over variables of the $n$ components that constrain the composition. It is not a designer's task to write the context-in components nor their sum. As they can be automatically generated (thanks to Definitions 2 and 4), the designer only specifies the components and the synchronization.

Recall that the four context-in components have the same set of variables $\operatorname{Var}_{A}=$ $\{c o, l w, r w, s e\}$. The wipers system $W S_{A}=\|_{c s w_{A}}\left(C O_{A}, S E_{A}, L W_{A}, R W_{A}\right)$ equals to $C O_{A}^{c} \uplus S E_{A}^{c} \uplus L W_{A}^{c} \uplus R W_{A}^{c}$.

\section{Synchonized Component-based Systems Refinement}

In this section, we first recall the refinement semantics given in $[8,9]$ for classical one component transition systems. A refinement relation in the style of the Milner-Park simulation relation between abstract and concrete transition systems is given. Second, we want these semantics to fit with the synchronized component-based systems. For that we define a refinement relation between transition systems which is weaker than the refinement relation introduced in $[8,9]$. Nevertheless, this weak refinement allows us to ensure the refinement of the whole system in a compositional way, unlike the refinement defined in $[8,9]$. We begin by an example before giving formal definitions.

Consider a refinement of the wind-screen wipers system, still composed of four components: $C O_{R}, S E_{R}, L W_{R}$ and $R W_{R}$. In $C O_{R}$ the speed choice seCo is per- 


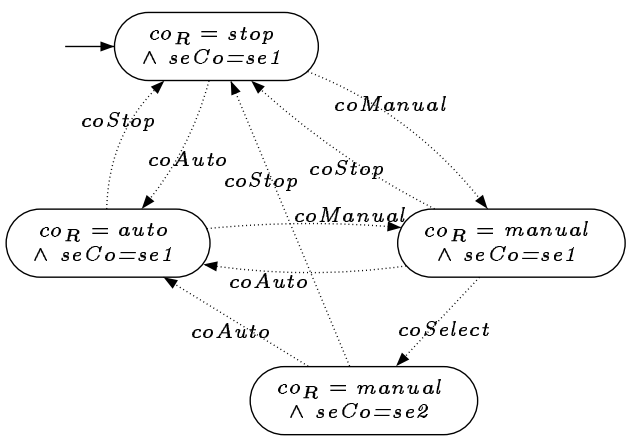

Figure 6: $C O_{R}$

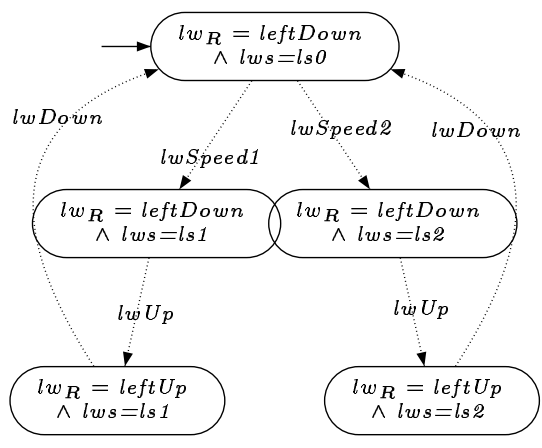

Figure 7: $L W_{R}$

formed in the manual mode: seCo=se1 for speed 1 or seCo=se2 for speed 2 (see Fig. 6). In both $L W_{R}$ and $R W_{R}$ the wind-screen wipers speed is taken into account. For $L W_{R}$ a new variable lws (for left wiper speed) is introduced with $\mathbb{D}($ lws $)=\{$ ls0, $l s 1, l s 2\}$ for respectively, no speed, speed 1 or speed 2 (see Fig. 7). Idem for $R W_{A}$. In the abstract system $S E_{A}$, there are two rain levels. We observe in $S E_{R}$ both limits of the tiny-rain level $\left(s e_{R}=\right.$ maxTiny $)$ and the strong-rain level $\left(s e_{R}=\right.$ minStrong $)$.

The authorized behaviours of the refined wipers system $W S_{R}$ are described in the synchronization $c s w_{R}$. We still have the old synchronized behaviours, i.e. left and right wipers must move together ((lwDown, rwDown) and ((lwUp, rwUp)), the control lever and the rain sensor change only if the wipers are down (coManual, coStop, seTiny, seStrong), etc. In addition, some of the new transitions are also synchronized. For example, the speed selection in the control lever manual mode can happen only if the wipers are down (coSelect). The wipers change their speeds simultaneously ((lwSpeed1,rwSpeed1) and (lwSpeed2,rwSpeed2)). In the control lever auto mode, the tiny-rain detection is synchronized with the level 1 of the wipers speeds ((seMaxTiny,lwSpeed1,rwSpeed1)), and the strong-rain detection is synchronized with the level 2 of the wipers speeds ((seMinStrong,lwSpeed2,rwSpeed2)).

\subsection{Basic Transition Systems Refinement}

Let $S A=<Q_{A}, Q_{0 A}, E_{A}, T_{A}, l_{A}>$ be an abstract transition system over $\operatorname{Var}_{A}$ and $S R=<Q_{R}, Q_{0 R}, E_{R}, T_{R}, l_{R}>$ a concrete transition system over $\operatorname{Var}_{R}$. In this section, some basic definitions about transition systems refinement are given. The syntactic concepts of the refinement are the following. Refinement introduces new 
transition labels, so $E_{A} \subseteq E_{R}$. Refinement introduces new variables and renames abstract ones, so $\operatorname{Var}_{A} \cap \operatorname{Var}_{R}=\varnothing$.

Let $G I$ be a formula over $S P_{A} \cup S P_{R} \cup S P^{\prime}$ where $S P_{A}$ is over $\operatorname{Var}_{A}, S P_{R}$ is over $\operatorname{Var}_{R}$, and $S P^{\prime} \stackrel{\text { def }}{=}\left\{X_{A}=X_{R}\right\}$ with $X_{A} \in V a r_{A}$ and $X_{R} \in V a r_{R}$ and $\mathbb{D}\left(X_{A}\right)=\mathbb{D}\left(X_{R}\right)$. $G I$ is commonly known as a gluing invariant linking variables of the abstract and concrete systems. A binary relation $\mu \subseteq Q_{R} \times Q_{A}$ allows us to express this link between the states of two transition systems $[8,9]$.

\section{Definition 6 (Gluing Relation)}

Let $G I$ be a gluing invariant between $S R$ and $S A$. The states $q_{R} \in Q_{R}$ and $q_{A} \in Q_{A}$ are glued, written $q_{R} \mu q_{A}$, iff $l_{R}\left(q_{R}\right) \wedge G I \Rightarrow l_{A}\left(q_{A}\right)$.

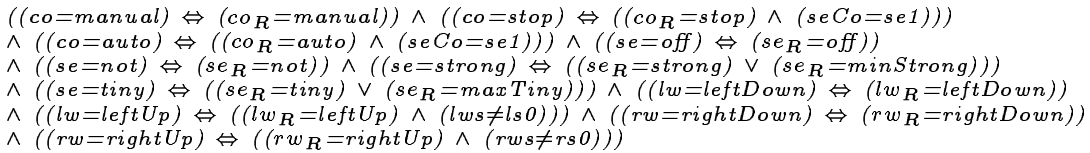

Figure 8: Gluing invariant $G I_{W}$

The gluing invariant $G I_{W}$ linking variables of the refined system $W S_{R}$ and the abstract system $W S_{A}$ is given in Fig. 8. The abstract variable co from $C O_{A}$ is linked with the concrete variables $c o_{R}$ and $s e C o$. If $c o=$ manual then $c o_{R}=$ manual, if $c o=$ stop then $c o_{R}=$ stop and $s e C o=s e 1$, and if $c o=$ auto then $c o_{R}=$ auto and $s e C o=s e 1$. We have the same kind of links between the variables of $S E_{A}, L W_{A}, R W_{A}$ and respectively, $S E_{R}, L W_{R}, R W_{R}$.

The semantic concepts of the refinement are the following.

1. In order to describe the refinement, we keep the transitions of $S_{R}$, the labels of which are in $E_{A}$ (i.e. labelled by the "old" labels) and we consider the new transitions introduced during the refinement design (i.e. labelled in $E_{R} \backslash E_{A}$ ) as being non-observable; they are labelled by $\tau$ and called $\tau$-transitions. Each portion of path containing $\tau$-transitions must end by a transition labelled in $E_{A}$. Therefore, the transition refinement is either a strict refinement or a stuttering refinement (see Fig. 9).

2. In order to avoid livelocks, new transitions should not take control forever. So, the paths containing infinite sequences of $\tau$-transitions are forbidden.

3. Moreover, new transitions should not introduce deadlocks. 

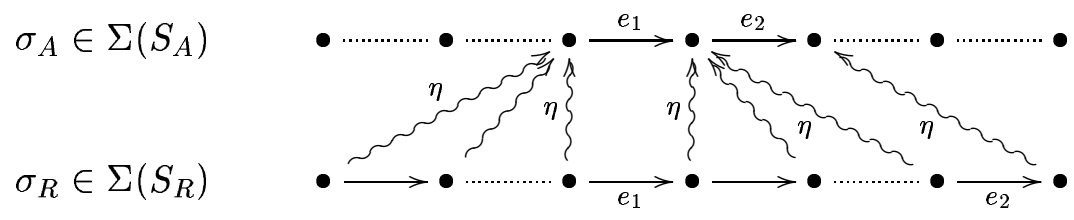

Figure 9: Path refinement

The refinement relation $\eta$ is defined in $[8,9]$ as a restriction of $\mu$ that verifies the previous concepts.

\section{Definition 7 (Strict Refinement Relation [8, 9])}

Let $S A$ and $S R$ be two transition systems, and $e$ be a label from $E_{A}$. We define the refinement relation $\eta$ as the greatest binary relation included into $\mu$ and satisfying the following conditions:

1) strict transition refinement

$\left(q_{R} \eta q_{A} \wedge q_{R} \stackrel{e}{\rightarrow} q_{R}^{\prime} \in T_{R}\right) \Rightarrow \exists q_{A}^{\prime} .\left(q_{A} \stackrel{e}{\rightarrow} q_{A}^{\prime} \in T_{A} \wedge q_{R}^{\prime} \eta q_{A}^{\prime}\right)$,

2) stuttering transition refinement

$\left(q_{R} \eta q_{A} \wedge q_{R} \stackrel{\tau}{\rightarrow} q_{R}^{\prime} \in T_{R}\right) \Rightarrow\left(q_{R}^{\prime} \eta q_{A}\right)$,

3) lack of new deadlocks

$\left(q_{R} \eta q_{A} \wedge q_{R} \nrightarrow_{R}\right) \Rightarrow\left(q_{A} \nrightarrow_{A}\right)^{1}$,

4) lack of $\tau$-divergence

$q_{R} \eta q_{A} \Rightarrow \neg\left(q_{R} \stackrel{\tau}{\rightarrow} q_{R}^{\prime} \stackrel{\tau}{\rightarrow} q_{R}^{\prime \prime} \stackrel{\tau}{\rightarrow} \ldots \stackrel{\tau}{\rightarrow} \ldots\right)$,

5) external non-determinism preservation

$\left(q_{A} \stackrel{e}{\rightarrow} q_{A}^{\prime} \in T_{A} \wedge q_{R} \eta q_{A}\right) \Rightarrow \exists q_{R}^{\prime}, q_{R}^{\prime \prime}, q_{A}^{\prime \prime} .\left(q_{R}^{\prime} \eta q_{A} \wedge q_{R}^{\prime} \stackrel{e}{\rightarrow} q_{R}^{\prime \prime} \in T_{R} \wedge q_{A} \stackrel{e}{\rightarrow}\right.$ $\left.q_{A}^{\prime \prime} \in T_{A} \wedge q_{R}^{\prime \prime} \eta q_{A}^{\prime \prime}\right)$.

We say that $S R$ refines $S A$, written $S R \sqsubseteq S A$, when the conditions above are verified between the states of $S R$ and $S A$ i.e. $S R \sqsubseteq S A \Leftrightarrow \forall q_{R} .\left(q_{R} \in Q_{R} \Rightarrow \exists q_{A} .\left(q_{A} \in\right.\right.$ $\left.\left.Q_{A} \wedge q_{R} \eta q_{A}\right)\right)$.

It has been shown in [8] that $\eta$ is a kind of $\tau$-simulation. It is well-known that a simulation can be computed iteratively for finite state systems. We have an algorithm based on a depth-first search enumeration of the reachability graph of the refined system. Its order is $O(|S R|)$ where $|S R|=\left|Q_{R}\right|+\left|T_{R}\right|$.

\footnotetext{
${ }^{1}$ We note $q \nrightarrow$ when $\forall q^{\prime}, e .\left(q^{\prime} \in Q \wedge e \in E \Rightarrow\left(q \stackrel{e}{\rightarrow} q^{\prime}\right) \notin T\right)$.
}

$\mathrm{RR} \mathrm{n}^{\circ} 4862$ 


\subsection{Compositional Component-based Systems Refinement}

In this section, the refinement semantics is fitted with synchronized componentbased systems. We define a refinement relation weaker than the refinement relation presented in Section 3.1, and we clarify how $\tau$ covers the new transition labels. Then we give a compositionality theorem allowing us to compositionally ensure the refinement of the whole system from the refinement of its components.

We have shown in [21] that $\eta$ is too strong to verify a component-based systems refinement. The problem is that some new deadlocks in the context-in components, could cause the refinement verification of a context-in component to fail whereas the refinement of the whole system is verified. That is why, we introduce another relation, called the weak refinement relation and written $\eta_{f}$. This relation is fitted to ensure the refinement verification in this case. The relation $\eta_{f}$ uses the set $D \subseteq Q_{R}$ of new deadlocks which is built during the refined system exploration.

\section{Definition 8 (Weak Refinement Relation)}

Let $S A$ and $S R$ be two transition systems, and $e$ be a label from $E_{A}$. Let $D \subseteq Q_{R}$ (Initially $D=\varnothing$ ) be the set of new deadlocks. We define the weak refinement relation $\eta_{f}$ as the greatest binary relation included into $\mu$ and satisfying the conditions $\mathbf{1}$ ), 2), 4) and 5) of Definition 7 and the following condition:

\section{3') old or new deadlocks}

$\left(q_{R} \eta_{f} q_{A} \wedge q_{R} \nrightarrow_{R}\right) \Rightarrow\left(\left(q_{A} \nrightarrow A\right) \vee\left(\left(q_{A} \stackrel{e}{\rightarrow} q_{A}^{\prime} \in T_{A}\right) \Rightarrow\left(q_{R} \in D\right)\right)\right)$.

We say that $S R$ weakly refines $S A$, written $S R \sqsubseteq_{D} S A$, when the conditions above are verified between the states of $S R$ and $S A$ i.e. $S R \sqsubseteq_{D} S A \Leftrightarrow \forall q_{R} \cdot\left(q_{R} \in Q_{R} \Rightarrow\right.$ $\left.\exists q_{A} \cdot\left(q_{A} \in Q_{A} \wedge q_{R} \eta_{f} q_{A}\right)\right)$.

The relation $\eta_{f}$ can be computed by an iterative algorithm with a complexity order in $O(|S R|)$ too. It is easy to see that the strict refinement relation $\eta$ implies the weak refinement relation $\eta_{f}$ when $D=\varnothing$, and vice versa. Indeed, condition $3^{\prime}$ ) of Definition 8 is verified for the old deadlocks, so $D$ remains empty.

\section{Property 1}

Let $S A$ and $S R$ be two transition systems. Let $D \subseteq Q_{R}$ be the set of new deadlocks. We have $(S R \sqsubseteq S A) \Leftrightarrow\left(S R \sqsubseteq_{D} S A \wedge D=\varnothing\right)$.

We have to clarify how $\tau$ covers new transition labels when we deal with synchronized component-based systems. Indeed, we want to avoid the refinement verification failure when an old transition and a new one are synchronized. The problem is then to decide whether this synchronized transition has to be covered by $\tau$ or not. 
Our approach is the following. On the one hand, the old transition label must be saved if it is a label of the component being considered. On the other hand, the transition must be kept out if its label is not a label of the considered component. We define a context-in $\tau$-component to be a context-in component covered by $\tau$. The first step of Definition 9 deletes some transitions and their labels, and the second step covers by $\tau$ the remaining transition labels.

\section{Definition 9 (Context-in $\tau$-component)}

Let $S A_{1}, S A_{2}, S R_{1}$ and $S R_{2}$ be four components. Let $S R_{1}^{c}$ be a context-in component. The context-in $\tau$-component $S R_{1}^{\tau}$ is the tuple $<Q_{R 1}^{\tau}, Q_{R 01}^{\tau}, E_{R 1}^{\tau}, T_{R 1}^{\tau}, l_{R 1}^{\tau}>$ where:

- $Q_{R 1}^{\tau}=Q_{R 1}^{c}$,

- $Q_{R 01}^{\tau}=Q_{R 01}^{c}$

- $l_{R 1}^{\tau}\left(\left(q_{1}, q_{2}\right)\right)=l_{R 1}^{c}\left(\left(q_{1}, q_{2}\right)\right)$,

- $T_{R 1}^{\tau}=T_{R 1}^{c} \backslash\left\{\left(\left(q_{1}, q_{2}\right),\left(e_{1}, e_{2}\right),\left(q_{1}^{\prime}, q_{2}^{\prime}\right)\right) \mid\left(\left(q_{1}, q_{2}\right),\left(e_{1}, e_{2}\right),\left(q_{1}^{\prime}, q_{2}^{\prime}\right)\right) \in\right.$

$\left.T_{R 1}^{c} \wedge e_{1} \in E_{R 1} \backslash E_{A 1} \wedge e_{2} \in E_{A 2}\right\}$

- $E_{R 1}^{\tau}=E_{R 1}^{c} \backslash\left\{\left(e_{1}, e_{2}\right) \mid\left(e_{1}, e_{2}\right) \in E_{R 1}^{c} \wedge e_{1} \in E_{R 1} \backslash E_{A 1} \wedge e_{2} \in E_{A 2}\right\}$.

Then, the elements of $E_{R 1}^{\tau}$ are covered as follows ${ }^{2}$ :

- if $\left(e_{1},-\right) \in E_{R 1}^{\tau}$ and $e_{1} \in E_{R 1} \backslash E_{A 1}$ then $\left(e_{1},-\right) \backslash \tau$,

- if $\left(e_{1}, e_{2}\right) \in E_{R 1}^{\tau}, e_{1} \in E_{R 1} \backslash E_{A 1}$ and $e_{2} \in E_{R 2} \backslash E_{A 2}$ then $\left(e_{1}, e_{2}\right) \backslash \tau$,

- if $\left(e_{1}, e_{2}\right) \in E_{R 1}^{\tau}, e_{1} \in E_{A 1}$ and $e_{2} \in E_{R 2} \backslash E_{A 2}$ then $\left(e_{1}, e_{2}\right) \backslash\left(e_{1},-\right)$.

Our first result is a compositional refinement verification theorem. This theorem links the separate context-in components weak refinements with the componentbased system refinement. It is based on deadlocks reduction. A state inducing a new deadlock in a component does not induce a deadlock in the whole system if there exists another component in which this state is not a deadlock state.

\section{Definition 10 (New Deadlocks Reduction)}

Let $S A_{1}, S A_{2}, S R_{1}$ and $S R_{2}$ be four components such that $S R_{1}^{\tau} \sqsubseteq D_{1} S A_{1}^{c}$ and $S R_{2}^{\tau} \sqsubseteq_{D_{2}} S A_{2}^{c}$. Let Synch and $S y n c h^{\prime}$ be two respective synchronizations. The set $D_{1,2} \subseteq Q_{R 1}^{\tau} \cup Q_{R 2}^{\tau}$ of states producing new deadlocks during the weak refinement of $S R_{1} \|_{\text {Synch }^{\prime}} S R_{2}$ is defined by:

$$
D_{1,2} \stackrel{\text { def }}{=}\left(D_{1} \cap D_{2}\right) \cup\left(D_{1} \backslash Q_{R 2}^{\tau}\right) \cup\left(D_{2} \backslash Q_{R 1}^{\tau}\right)
$$

We can compute the set $D_{1, \ldots, n}$ containing the new deadlocks during the weak refinement verification of $\|_{S y n c h^{\prime}}\left(S R_{1}, \ldots, S R_{n}\right)$. It is an associative computation:

\footnotetext{
${ }^{2}$ We note $e_{1} \backslash e_{2}$ the relabelling of $e_{1}$ by $e_{2}$.
} 
$D_{1, \ldots, n}=D_{(1, \ldots, n-1), n}=D_{1,(2, \ldots, n)}$. Then, Property 1 allows us to decide the strict refinement of the whole system.

\section{Theorem 1 (Refinement of a Synchronized Component-based System)}

Let $S A_{1}, \ldots, S A_{n}$, and $S R_{1}, \ldots, S R_{n}$ be $n$ abstract and refined components. Let Synch and Synch' be two respective synchronizations.

$$
\begin{aligned}
\|_{\text {Synch }^{\prime}} & \left(S R_{1}, \ldots, S R_{n}\right) \sqsubseteq \|_{S y n c h}\left(S A_{1}, \ldots, S A_{n}\right) \text { iff } \\
& -\forall i .1 \leq i \leq n \Rightarrow S R_{i}^{\tau} \sqsubseteq_{i} S A_{i}^{c} \\
& -D_{1, \ldots, n}=\varnothing
\end{aligned}
$$

A proof is given in [19].

This theorem provides a compositional refinement verification algorithm. This algorithm is based on the computation of the relation $\eta_{f}$ for each context-in component $S R_{i}^{\tau}$ which complexity order is $O\left(\left|S R_{i}^{\tau}\right|\right)$. The complexity of this refinement algorithm is $O\left(\left|S R_{1}^{\tau}\right|+\cdots+\left|S R_{n}^{\tau}\right|\right)$ but it can be iteratively computed. The greatest memory space used by this algorithm computation is $\max _{i=1}^{n}\left(\left|S R_{i}^{\tau}\right|\right)$, at most.

For our example, we built the refined context-in components. Figure 10 shows the refined context-in component $S E_{R}^{c}$. This context-in component only describes behaviours of $S E_{R}$ under $c s w_{R}$ that are either old transitions (seNot, seTiny, seStrong or (seOff,coStop), (seNot,coAuto)) or new transitions (seMaxTiny, seMinStrong or (seMaxTiny,lwSpeed1,rwSpeed1), (seMinStrong,lwSpeed2,rwSpeed2)).

By Definition 5, we have $W S_{R}=C O_{R}^{c} \uplus S E_{R}^{c} \uplus L W_{R}^{c} \uplus R W_{R}^{c}$ over the set of variables $\operatorname{Var}_{R}=\left\{c o_{R}, s e C o, l w_{R}, l w s, r w_{R}, r w s, s e_{R}\right\}$. The weak refinement is verified for each context-in component. We have $S E_{R}^{c} \sqsubseteq_{D_{S E}} S E_{A}^{c}$ where the set $D_{S E}$ contains two new deadlocks $q_{6}^{c}$ and $q_{8}^{c}$ (see Fig. 10). By Definition 10, we reduce $D_{S E}$ and we show that $D_{C O, S E, L W, R W}$ is empty. By Theorem 1, we conclude that the wipers system $W S_{R}$ refines $W S_{A}$.

The refinement relation provides a formal framework for verifying system properties. When the refinement is verified, most properties that have been verified on the abstract system are preserved on the refined one.

\section{Compositional Property Verification}

In this section, we propose a method to verify a class of safety properties on synchronized component-based systems. These properties can be expressed as the unreachability of a set of states. If this set is not reachable in the abstract system, then the 


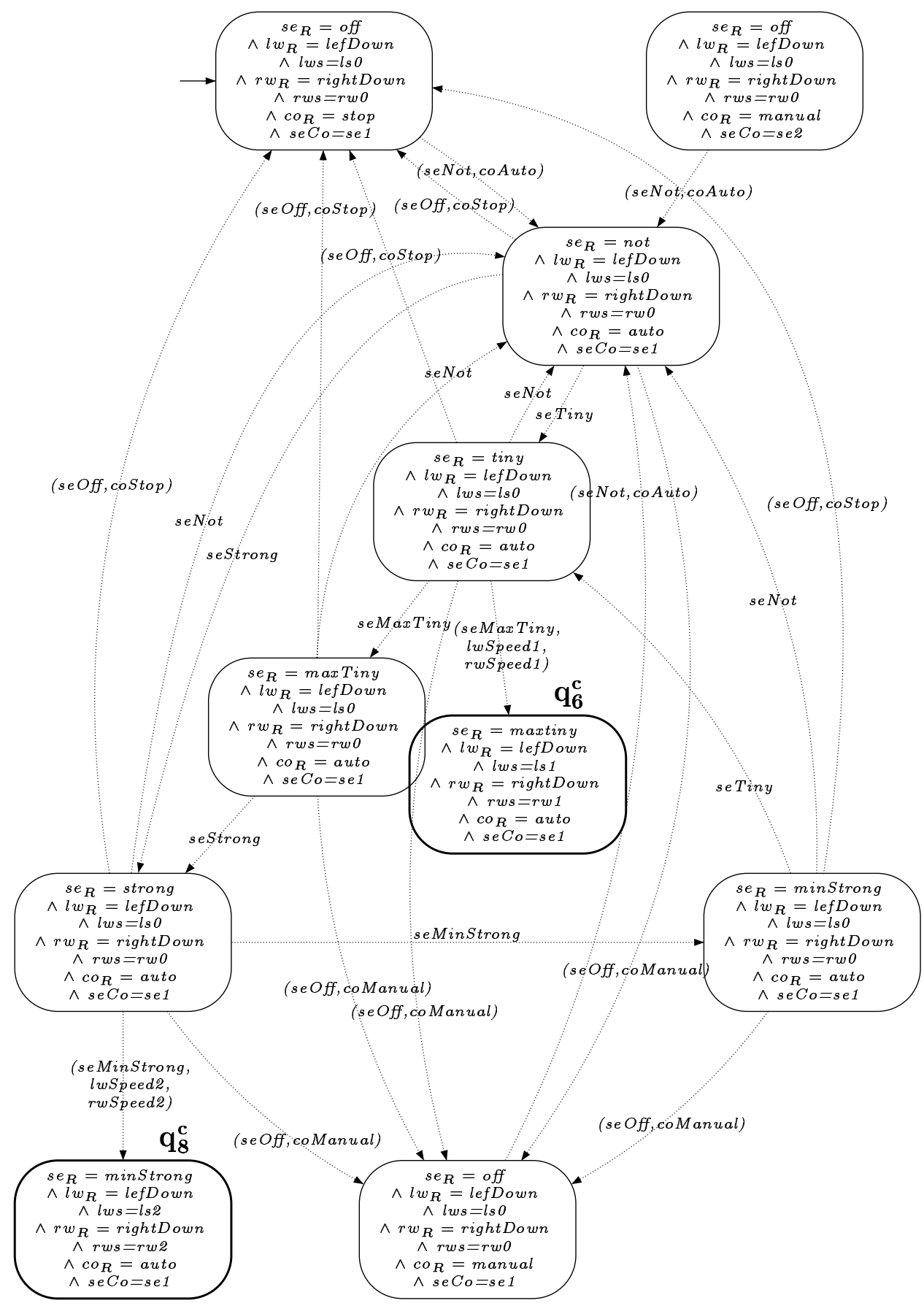

Figure 10: $S E_{R}^{c}$

$\mathrm{RR} \mathrm{n}^{\circ} 4862$ 
refinement relation ensures the unreachability of the corresponding set in the refined system.

Since we introduce new details in the refinement, the abstract system is usually small in number of states while the refined system is likely to be very large. We want to exploit the refinement approach enriched by a component paradigm. It allows us to avoid the model-checking blow-up by verifying safety properties in a compositional way.

The method we propose here is based on Definition 5. The idea is to verify the reachability among the context-in components $S_{i}^{c}$ instead of exploring the whole system $\|_{\text {Synch }}\left(S_{1}, \ldots, S_{n}\right)$. Actually, the state space and the transition relation of a context-in component are less important than the entire system ones. We reduce the number of possible behaviours during the system exploration, and, consequently, we postpone the state space explosion problem.

Let $\|_{\text {Synch }}\left(S_{1}, \ldots, S_{n}\right)$ be a synchronized parallel composition under Synch. The reachability problem for $\|_{S y n c h}\left(S_{1}, \ldots, S_{n}\right)$ is the following decision problem.

Input: $n$ context-in components $S_{1}^{c}, \ldots, S_{n}^{c}$ and a target state $q_{t}^{c}$ s.t. $q_{t}^{c} \in \bigcup_{i=1}^{n} Q_{i}^{c}$. Reachability problem (RP): Determine whether $q_{t}^{c}$ is in $\left\{q^{c} \mid q^{c} \in \bigcup_{i=1}^{n} Q_{i}^{c} \wedge\right.$ $\left.\exists q_{0}^{c} .\left(q_{0}^{c} \in \bigcup_{i=1}^{n} Q_{0 i}^{c} \wedge \exists w .\left(w \in\left(\bigcup_{i=1}^{n} E_{i}^{c}\right)^{*} \wedge\left(q_{0}^{c}, w, q^{c}\right) \in\left(\bigcup_{i=1}^{n} T_{i}^{c}\right)^{*}\right)\right)\right\}$, where $\left(\bigcup_{i=1}^{n} T_{i}^{c}\right)^{*}$ is the reflexive and transitive closure of the transition relation $\bigcup_{i=1}^{n} T_{i}^{c}$.

\section{Theorem 2 (RP for a Synchronized Component-based System)}

There exists an algorithm to decide the reachability problem for a synchronized component-based system $\|_{\text {Synch }}\left(S_{1}, \ldots, S_{n}\right)$ whose complexity order is in $O\left(\left|S_{1}^{c}\right|+\right.$ $\left.\cdots+\left|S_{n}^{c}\right|\right)$.

In practice, to verify the reachability of a target state $q_{t}^{c}$ in a context-in component, we apply the backward-reachability analysis from $q_{t}^{c}$. During this exploration, the set $Q_{D}$ of the deadlock states is built. If no initial state is reachable, we choose another context-in component, and, often, a new target state among the deadlock states (in $Q_{D}$ ), in order to continue the reachability analysis from this state. We can choose a new target, since the state $q_{t}^{c}$ is reachable from this new state. We stop when either an initial state $q_{0}^{c} \in \bigcup_{i=1}^{n} Q_{0 i}^{c}$ is reached or exploration of all possible choices is already done.

The verification algorithm below formally presents the compositional reachability analysis. 
possibility to have the variable reach equal to true (line 35) is to reach an initial state.

For our running wind-screen wipers example, we want some states to be forbidden. For instance, states where the wipers system can move only one of the wind-screen wipers, must be unreachable. Then we exploit the refinement to ensure this property for the refined system.

Consider $q_{t}^{c}$, a state such that $l\left(q_{t}^{c}\right) \stackrel{\text { def }}{=}(l w=$ leftDown $\wedge r w=$ right $U p \wedge c o=$ manual $\wedge s e=o f f)$. We want to ensure the unreachability of the state $q_{E}^{c}$, written $\square\left(\neg q_{t}^{c}\right)$ in $P L T L$. By Algorithm 1, it is possible to show that $q_{t}^{c}$ is not reachable in the abstract system $W S_{A}$. As the system $W S_{R}$ refines the system $W S_{A}$ (as explained in Section 3), the refined system states corresponding to $q_{t}^{c}$ by the gluing invariant $G I_{W}$ are unreachable too.

\section{SynCo: a Tool Verifying the Synchronized Component- based Systems Refinement}

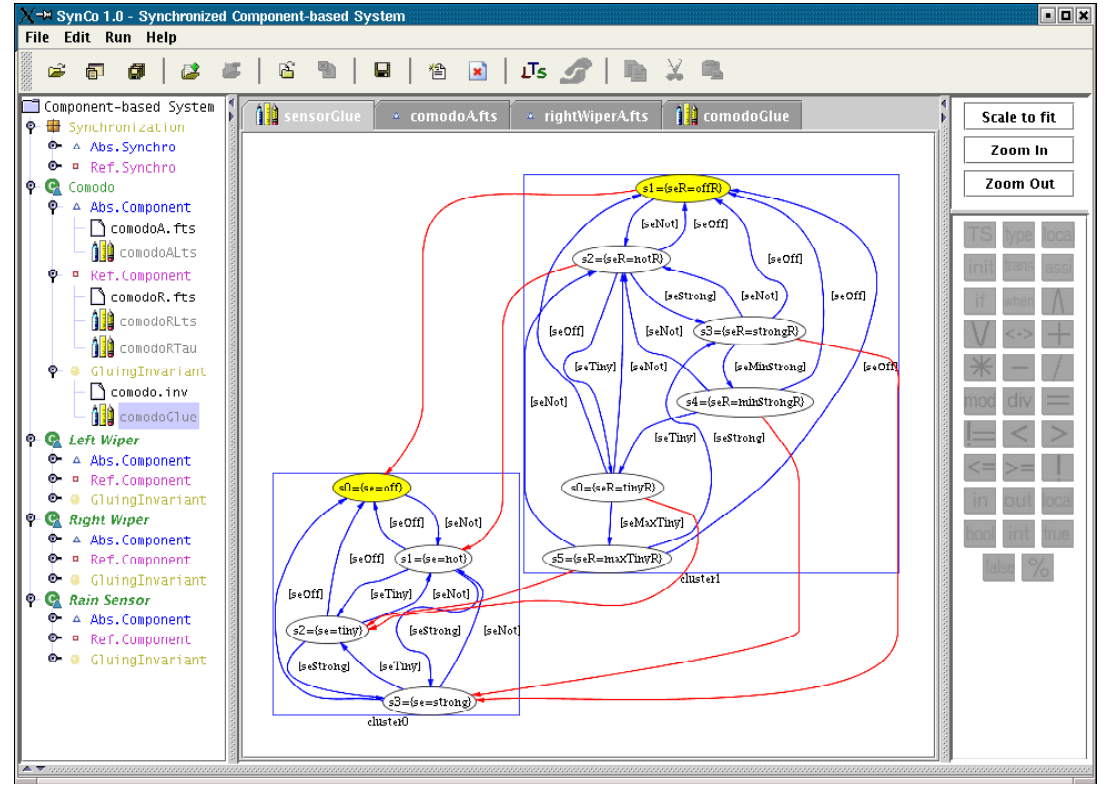

Figure 11: The tool SynCo 
Theorem 1 in Section 3 gives the required conditions to ensure the strict refinement of a whole system from the weak refinements of its synchronized components, and vice versa. This way, we have an algorithm to verify the strict refinement of a synchronized component-based system. The most important advantage of this refinement verification is that it is not necessary to build the whole system to verify the refinement.

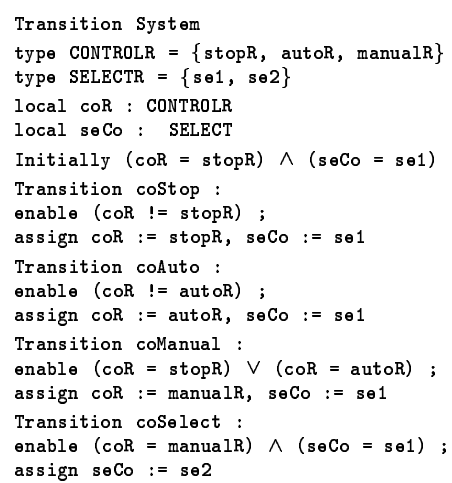

Figure 12: $C O_{R}$ in $\mathrm{STeP}$

We are developing SynCo (for Synchronized Component-based System Analyser $)^{3}$; a tool implementing with Java the synchronized component refinement verification (Figure 11 show a capture of its interface). We specify the components as transition systems using the STeP's Fair Transition Systems syntax [23, 10]. Each transition is specified with its activation condition (enable) and its assignments (assign). Figure 12 gives the $\mathrm{STeP}$ specification of the component $C O_{R}$.

The context-in components are built automatically from both STeP and synchronization specifications. Each component or each context-in component can be visualised as an automaton using the Java package Grappa from the toolkit Graphviz ${ }^{4}$.

Theorem 1 is implemented in SynCo to verify the refinement of a synchronized component-based system. We compute the relation $\eta_{f}$ for each context-in $\tau$-component using a kind of depth-first search enumeration of the reachability graph of this context-in $\tau$-component. Then, we conclude about the refinement of the whole system. Figure 11 shows the relation $\eta_{f}$ between a refined component and an abstract component.

The refinement verification of a simple system can be done with SynCo too. We assume that the simple system is the one component of a synchronized componentbased system. Obviously, the new deadlocks set is empty by Property 1.

With this tool the refinement verification of the wind-screen wipers system and of an industrial robot has been done. Thanks to SynCo we can measure the efficiency of our method. Tables 13 and 14 give the results of our tests. For the wind-screen wipers system $W S_{R}$ both the states and the transitions are decreased by a third between the whole system and the greatest context-in component (see Fig. 13). We

\footnotetext{
${ }^{3}$ SynCo home page: http://lifc.univ-f comte.fr/ lanoix/synco.html

${ }^{4}$ Graphviz official page: http://www.research.att.com/sw/tools/graphviz
}

$\mathrm{RR} \mathrm{n}^{\circ} 4862$ 


\begin{tabular}{|c||c|c|c|c||c|}
\hline & $C O_{R}^{c}$ & $S E_{R}^{c}$ & $L W_{R}^{c}$ & $R W_{R}^{c}$ & $W S_{R}$ \\
\hline$\left|Q_{R}\right|$ & 8 & 10 & 18 & 18 & 20 \\
\hline$\left|T_{R}\right|$ & 17 & 25 & 20 & 20 & 47 \\
\hline$|S R|$ & 25 & 35 & 38 & 38 & 67 \\
\hline
\end{tabular}

\begin{tabular}{|c||c|c|c||c|}
\hline & $C L_{R}^{c}$ & $L I_{R}^{c}$ & $H A_{R}^{c}$ & $R O_{R}$ \\
\hline$\left|Q_{R}\right|$ & 19 & 13 & 19 & 25 \\
\hline$\left|T_{R}\right|$ & 21 & 13 & 20 & 40 \\
\hline$|S R|$ & 40 & 26 & 39 & 65 \\
\hline
\end{tabular}

Figure 13: The wipers system

Figure 14: The robot

have similar results in Fig. 14 for the industrial robot $R O_{R}$ moving pieces thanks to a clip $C L_{R}$, a handle $H A_{R}$ and a lift $L I_{R}$. We plan to test $S y n C o$ on more complex examples.

\section{Conclusion and Perspectives}

Compositional design and refinement paradigms are very convenient to specify and to verify large embedded reactive systems such as automatic trains, car driving assistance systems or communication protocols. In this paper, we propose a technique to take advantages of both paradigms. This technique is based on a specific notion of refinement relation defined in the style of Milner-Park simulation relation between concrete and abstract finite transition systems.

The contributions of this paper are the following. On the one hand, we want the refinement paradigm to be compatible with the compositional specification. For that, we propose a new compositional specification method that allows us to ensure the refinement of the whole system from the refinement of its context-in components, and vice versa. Although context-in components seem comparable with the whole system, they are generally smaller than the whole system. On the other hand, we want to avoid the model-checking blow-up by verifying a class of safety properties in a compositional way. For that, we give an algorithm to ensure the unreachability of a set of states of a synchronized component-based system. Moreover, this algorithm is very interesting when combined with the compositional refinement verification algorithm.

A tool that implements the compositional refinement verification has been implemented in Java. This tool, SynCo, allows us to measure the efficiency of our approach, and to test it to real industrial examples. We are currently working on extending this tool to incorporate the compositional reachability algorithm. In addition, we are going to use the strong dependency analysis of [22] as heuristics to efficiently choose a pair $\left(q_{i}^{c}, S_{i}^{c}\right)$. As far as we know, other compositional tools also implement a reachability compositional verification (see for instance [15, 22]). How- 
ever, they were designed for a very different purpose than SynCo and, thus, do not implement compositional refinement verification.

One of the consequences of the composition theorem in [25] is the limitation of the compositional verification. It has been shown that the composition theorem fails for very simple parallel operators if there is a property expressing that "there is a path such that all the nodes of the path have a property $p$ ". Moreover, we have established in [14] that the strict refinement relation preserves the abstract system PLTL properties, i.e. safety and liveness properties because of Conditions 3) and 4) of Definition 7 . This preservation partially avoids the combinatorial explosion problem. Indeed, a property proof on an abstract system can be reused as a hypothesis for its verification on the refined system. That is why we are interested in a compositional verification of $P L T L$ properties.

More generally, we are going to use the works on property preservation [14], modular verification [24, 17], and dynamic property refinement [6, 7] in the framework of the component-based system specification and refinement presented in this paper. We hope this will allow us to verify a large spectrum of properties in a compositional way.

\section{Acknowledgement}

We are very grateful to Steve Campos and Caroline Lasalle for their participation in the development of SynCo.

\section{References}

[1] J.-R. Abrial. The B Book. Cambridge University Press - ISBN 0521-496195, 1996.

[2] J.-R. Abrial. Constructions d'automatismes industriels avec B. In Congrès $A F A D L$, ONERA-CERT - Toulouse, France, May 1997. Invited lecture.

[3] J.-R. Abrial. Discrete system models. Version 1.1, February 2002.

[4] J.-R. Abrial and L. Mussat. Introducing dynamic constraints in B. In Second Conference on the B method, France, volume 1393 of LNCS, pages 83-128. Springer Verlag, April 1998.

[5] P. Behm, P. Desforges, and J.M. Meynadier. MÉTÉOR: An industrial success in formal development. In Second conference on the B method, volume 1393 of

$\mathrm{RR} \mathrm{n}^{\circ} 4862$ 
Lecture Notes in Computer Science, Montpellier, France, April 1998. Springer Verlag. Invited lecture.

[6] F. Bellegarde, C. Darlot, J. Julliand, and O. Kouchnarenko. Reformulate dynamic properties during B refinement and forget variants and loop invariants. In Proc. First Int. Conf. ZB'2000, York, Great Britain, volume 1878 of LNCS, pages 230-249. Springer-Verlag, September 2000.

[7] F. Bellegarde, C. Darlot, J. Julliand, and O. Kouchnarenko. Reformulation: a way to combine dynamic properties and $\mathrm{B}$ refinement. In In Proc. Int. Conf. Formal Method Europe'01, Berlin, Germany, volume 2021 of LNCS, pages 2-19. Springer Verlag, March 2001.

[8] F. Bellegarde, J. Julliand, and O. Kouchnarenko. Ready-simulation is not ready to express a modular refinement relation. In Proc. Int. Conf. on Fundamental Aspects of Software Engineering, FASE'2000, volume 1783 of LNCS, pages 266283. Springer-Verlag, April 2000.

[9] F. Bellegarde, J. Julliand, and O. Kouchnarenko. Synchronized parallel composition of event systems in $B$. In D. Bert, J. P. Bowen, M. C. Henson, and $\mathrm{K}$. Robinson, editors, $Z B$ 2002 : Formal specification and development in $Z$ and $B$, volume 2272 of LNCS, pages 436-457. Springer-Verlag, 2002.

[10] N. Bjørner, A. Browne, M. Colón, B. Finkbeiner, Z. Manna, M. Pichora, H. B. Sipma, and T. E. Uribe. STeP - The Stanford Temporal Prover - Educational Release - User's Manual. Computer Science Department - Stanford University, Stanford, California 94305, july 1998.

[11] P. Bontron and M.-L. Potet. Automatic construction of validated B components from structured developments. In Proc. First Int. Conf. ZB'2000, York, Great Britain, volume 1878 of LNCS, pages 127-147. Springer-Verlag, September 2000.

[12] M. J. Butler. csp2B: A practical approach to combining CSP and B. Formal Aspects of Computing, 12:182-198, 2000.

[13] M. J. Butler and M. Waldén. Program Development by Refinement (Case Studies Using the B Method), chapter Parallel Programming with the B Method. Springer, 1999.

[14] C. Darlot, J. Julliand, and O. Kouchnarenko. Refinement preserves PLTL properties. In D. Bert, J. P. Bowen, S. C. King, and M. Walden, editors, ZB'2003: 
Formal Specification and Development in $Z$ and B, volume 2651 of $L N C S$, Turku, Finland, June 2003. Springer-Verlag.

[15] Hubert Garavel, Frédéric Lang, and Radu Mateescu. An overview of CADP 2001. Technical Report RT-254, INRIA, December 2001.

[16] J. Julliand, F. Bellegarde, and B. Parreaux. De l'expression des besoins à l'expression formelle des propriétés dynamiques. Technique et Science Informatiques, 18(7), 1999.

[17] J. Julliand, P-A. Masson, and H. Mountassir. Vérification par model-checking modulaire des propriétés dynamiques introduites en B. Technique et Science Informatiques, 20(7):927-957, 2001.

[18] J. Julliand, P.A. Masson, and H. Mountassir. Modular verification of dynamic properties for reactive systems. In International Workshop on Integrated Formal Methods (IFM'99), York, Great Britain, 1999.

[19] O. Kouchnarenko and A. Lanoix. Refinement and verification of synchronized component-based systems. INRIA Research Report, June 2003. To appear.

[20] L. Lamport. Specifying concurrent systems with TLA+. In Calculational System Design, Amsterdam, 1999. IOS Press.

[21] A. Lanoix. Raffinement de systèmes à composants synchronisés et leur vérification. Mémoire de DEA - UFR Sciences et Techniques - Université de FrancheComté, Septembre 2002.

[22] J. Lind-Nielsen, H. R. Andersen, H. Hulgaard, G. Behrmann, K. Kristoffersen, and K. G. Larsen. Verification of large state/event systems using compositionality and dependency analysis. Formal Methods in System Design, 18(1):5-23, January 2001.

[23] Z. Manna, N. Bjørner, A. Browne, E. Chang, M. Colón, Luca de Alfaro, H. Devarajan, A. Kapur, J. Lee, H. B. Sipma, and T. E. Uribe. STeP - the stanford temporal prover. Theory and Practice of Software Developpement, 915 of LNCS:793-794, May 1995.

[24] P.-A. Masson, H. Mountassir, and J. Julliand. Modular verification for a class of PLTL properties. In T. Santen W. Grieskamp and B. Stoddart, editors, 2nd international conference on Integrated Formal Methods, IFM 2000, volume 1945 of $L N C S$, pages 398-419. Springer-Verlag, November 2000.

$\mathrm{RR} \mathrm{n}^{\circ} 4862$ 
[25] A. Rabinovich. On compositional method and its limitations. Technical report, University of Edinburgh, Research Report EDI-INF-RR-0035, 2001.

[26] S. Schneider and H. Treharne. Communicating $B$ machines. In D. Bert, J. P. Bowen, M. C. Henson, and K. Robinson, editors, ZB 2002 : Formal specification and development in $Z$ and $B$, volume 2272 of LNCS, pages 416-435. SpringerVerlag, 2002.

[27] E. Sekerinski. Program Development by Refinement (Case Studies Using the B Method), chapter Production Cell. Springer, 1999.

[28] H. Treharne, J. Draper, and S. Schneider. Test case preparation using a prototype. In Second conference on the B method, LNCS 1393, pages 293-312, Montpellier, France, April 1998. Springer Verlag.

[29] H. Treharne and S. Schneider. Using a process algebra to control B OPERATIONS. In IFM'99 1st International Conference on Integrated Formal Methods, pages 437-457, York, 1999. Springer-Verlag. 


\section{A Proof of Theorem 1}

We prove that $S R_{1}\left\|_{S y n c h_{R}} S R_{2} \sqsubseteq S A_{1}\right\|_{S y n c h_{A}} S A_{2} \quad[R] \quad$ iff $\begin{cases}S R_{1}^{\tau} \sqsubseteq D_{1} S A_{1}^{c}, & {[a]} \\ S R_{2}^{\tau} \sqsubseteq D_{2} S A_{2}^{c}, & {[b]} \\ D_{12}=\varnothing . & {[c]}\end{cases}$

It can be extended to $n$ components.

For that, we show that items $[a],[b]$ and $[c]$ imply $[R]$ (see Section A.1). Then, we show that $[R]$ ) implies $[a],[b]$ and $[c]$ (see Section A.2).

Let $Q_{R}^{c}$ be the state set of $S R_{1} \|_{S y n c h_{R}} S R_{2}$, and $Q_{A}^{c}$ the state set of $S A_{1} \|_{S y n c h_{A}} S A_{2}$. By Definition 2, we have $Q_{R}^{c}=Q_{R 1}^{c} \cup Q_{R 2}^{c}$ and $Q_{A}^{c}=Q_{A 1}^{c} \cup Q_{A 2}^{c}$.

A.1 $[a] \wedge[b] \wedge[c] \Rightarrow[R]$

Let $M \stackrel{\text { def }}{=}\left\{\left(q_{R}^{c}, q_{A}^{c}\right) \mid\left(\left(q_{R}^{c} \in Q_{R 1}^{c} \wedge q_{A}^{c} \in Q_{A 1}^{c}\right) \vee\left(q_{R}^{c} \in Q_{R 2}^{c} \wedge q_{A}^{c} \in Q_{A 2}^{c}\right)\right) \wedge q_{R}^{c} \eta_{f} q_{A}^{c}\right\}$.

We show that $M$ verifies the conditions of the refinement relation $\eta$ (Definition 7 ) using Lemmas 1, 2, 3, 4 and 5 .

By $[c]$ we have $D_{12}=\varnothing$. As $D_{12}=\left(D_{1} \cap D_{2}\right) \cup\left(D_{1} \backslash Q_{R 2}^{c}\right) \cup\left(D_{2} \backslash Q_{R 1}^{c}\right)$ (Definition 10), we have $\left[c^{\prime}\right]:\left(D_{1} \cap D_{2}=\varnothing\right) \wedge\left(D_{1} \backslash Q_{R 2}^{c}=\varnothing\right) \wedge\left(D_{2} \backslash Q_{R 1}^{c}=\varnothing\right)$.

\section{Lemma 1 (Strict Transition Refinement Condition)}

Assume that $q_{R}^{c} \stackrel{\alpha}{\rightarrow} q_{R}^{c \prime}$ and $\alpha \in E_{R 1}^{c} \cup E_{R 2}^{c}$; we must prove that there exists $q_{A}^{c \prime}$ such that $q_{A}^{c} \stackrel{\alpha}{\rightarrow} q_{A}^{c \prime}$ and $q_{R}^{c \prime} \eta q_{A}^{c \prime}$.

Proof on the form of $\alpha$. There are three cases.

- $\alpha=\left(e_{1},-\right)$. By Definition 4, we have $\left(q_{R}^{c},\left(e_{1},-\right), q_{R}^{c \prime}\right) \in T_{R 1}^{c}, q_{R}^{c} \in Q_{R 1}^{c}$ and $q_{R}^{c \prime} \in Q_{R 1}^{c}$. Since $S R_{1}^{\tau} \sqsubseteq S A_{1}^{c}$, we obtain that there exists $q_{A}^{c}$ such that $q_{A}^{c} \in Q_{A 1}^{c}$ and $q_{R}^{c} \eta q_{A}^{c}$. By condition 1 for $S R_{1}^{\tau} \sqsubseteq S A_{1}^{c}$, there exists $q_{A}^{c \prime}{ }^{\prime}$ such that $q_{A}^{c \prime} \in Q_{A 1}^{c}$, $q_{A}^{c} \stackrel{\left(e_{1},-\right)}{\rightarrow} q_{A}^{c \prime}$ and $q_{R}^{c \prime} \eta q_{A}^{c}$. The states $q_{A}^{c}$ and $q_{A}^{c \prime}$ belong to $Q_{A}^{c}$ because of $Q_{A}^{c}=$ $Q_{A 1}^{c} \cup Q_{A 2}^{c}$.

- $\alpha=\left(-, e_{2}\right)$. By Definition 4, we have $\left(q_{R}^{c},\left(-, e_{2}\right), q_{R}^{c \prime}\right) \in T_{R 2}^{c}, q_{R}^{c} \in Q_{R 2}^{c}$ and $q_{R}^{c \prime} \in Q_{R 2}^{c}$. Since $S R_{2}^{\tau} \sqsubseteq S A_{2}^{c}$, we obtain that there exists $q_{A}^{c}$ such that $q_{A}^{c} \in Q_{A 2}^{c}$ and $q_{R}^{c} \eta q_{A}^{c}$. By condition 1 for $S R_{2}^{\tau} \sqsubseteq S A_{2}^{c}$, there exists $q_{A}^{c \prime}$ such that $q_{A}^{c \prime} \in Q_{A 2}^{c}$, $q_{A}^{c} \stackrel{\left(-, e_{2}\right)}{\rightarrow} q_{A}^{c \prime}$ and $q_{R}^{c} \eta q_{A}^{c \prime}$. The states $q_{A}^{c}$ and $q_{A}^{c \prime}$ belong to $Q_{A}^{c}$ because of $Q_{A}^{c}=$ $Q_{A 1}^{c} \cup Q_{A 2}^{c}$.

- $\alpha=\left(e_{1}, e_{2}\right)$. By Definition 4, we have $\left(q_{R}^{c},\left(e_{1}, e_{2}\right), q_{R}^{c \prime}\right) \in T_{R 1}^{c}, q_{R}^{c} \in Q_{R 1}^{c}$ and $q_{R}^{c \prime} \in Q_{R 1}^{c}$. Since $S R_{1}^{\tau} \sqsubseteq S A_{1}^{c}$, we obtain that there exists $q_{A}^{c}$ such that $q_{A}^{c} \in Q_{A 1}^{c}$

$\mathrm{RR} \mathrm{n}^{\circ} 4862$ 
and $q_{R}^{c} \eta q_{A}^{c}$. By condition 1 for $S R_{1}^{\tau} \sqsubseteq S A_{1}^{c}$, there exists $q_{A}^{c \prime}$ such that $q_{A}^{c \prime} \in Q_{A 1}^{c}$, $q_{A}^{c} \stackrel{\left(e_{1},-\right)}{\rightarrow} q_{A}^{c \prime}$ and $q_{R}^{c \prime} \eta q_{A}^{c}$. The states $q_{A}^{c}$ and $q_{A}^{c \prime}$ belong to $Q_{A}^{c}$ because of $Q_{A}^{c}=$ $Q_{A 1}^{c} \cup Q_{A 2}^{c}$.

\section{Lemma 2 (Stuttering Transition Refinement Condition)}

Assume that $q_{R}^{c} \stackrel{\tau}{\rightarrow} q_{R}^{c \prime}$; we must show that $q_{R}^{c \prime} \eta q_{A}^{c}$.

Proof on the form of labels covered by $\tau$. There are three cases.

- $\left(e_{1}^{\prime},-\right) \backslash \tau$. By Definition 4, we have $\left(q_{R}^{c},\left(e_{1}^{\prime},-\right) \backslash \tau, q_{R}^{c \prime}\right) \in T_{R 1}^{c}, q_{R}^{c} \in Q_{R 1}^{c}$ and $q_{R}^{c \prime} \in Q_{R 1}^{c}$. Since $S R_{1}^{\tau} \sqsubseteq S A_{1}^{c}$, there exists $q_{A}^{c}$ such that $q_{A}^{c} \in Q_{A 1}^{c}$ and $q_{R}^{c} \eta q_{A}^{c}$. By condition 2 for $S R_{1}^{\tau} \sqsubseteq S A_{1}^{c}$, we obtain that $q_{R}^{c} \eta^{\prime} q_{A}^{c}$.

- $\left(-, e_{2}^{\prime}\right) \backslash \tau$. by Definition 4, we have $\left(q_{R}^{c},\left(-, e_{2}^{\prime}\right) \backslash \tau, q_{R}^{c}{ }^{\prime}\right) \in T_{R 2}^{c}, q_{R}^{c} \in Q_{R 2}^{c}$ and $q_{R}^{c \prime} \in Q_{R 2}^{c}$. Since $S R_{2}^{\tau} \sqsubseteq S A_{2}^{c}$, there exists $q_{A}^{c}$ such that $q_{A}^{c} \in Q_{A 2}^{c}$ and $q_{R}^{c} \eta q_{A}^{c}$. By condition 2 for $S R_{2}^{\tau} \sqsubseteq S A_{2}^{c}$, we obtain that $q_{R}^{c} \eta^{\prime} q_{A}^{c}$.

- $\left(e_{1}^{\prime}, e_{2}^{\prime}\right) \backslash \tau$. By Definition 4, we have $\left(q_{R}^{c},\left(e_{1}^{\prime}, e_{2}^{\prime}\right) \backslash \tau, q_{R}^{c \prime}\right) \in T_{R 1}^{c}, q_{R}^{c} \in Q_{R 1}^{c}$ and $q_{R}^{c \prime} \in Q_{R 1}^{c}$. Since $S R_{1}^{\tau} \sqsubseteq S A_{1}^{c}$, there exists $q_{A}^{c}$ such that $q_{A}^{c} \in Q_{A 1}^{c}$ and $q_{R}^{c} \eta q_{A}^{c}$. By condition 2 for $S R_{1}^{\tau} \sqsubseteq S A_{1}^{c}$, we obtain that $q_{R}^{c \prime} \eta q_{A}^{c}$.

\section{Lemma 3 (Lack of New Deadlocks Condition)}

Assume that $q_{R}^{c} \nrightarrow$; we must prove that $q_{A}^{c} \nrightarrow$.

Proof on the structure of the state set. There are three cases.

- $q_{R}^{c} \in Q_{R 1}^{c} \backslash Q_{R 2}^{c}$. As we have $q_{R}^{c} \eta_{f} q_{A}^{c}$, condition 3 in $S R_{1}^{\tau} \sqsubseteq D_{1} S A_{1}^{c}$ gives:

- either $q_{A}^{c} \nrightarrow$ and we are done,

- or $q_{A}^{c} \stackrel{e}{\rightarrow}_{A} q_{A}^{c \prime}$ and $q_{R}^{c} \in D_{1}$. As $q_{R}^{c} \notin Q_{R 2}^{c}$, we have a contradiction with $D_{1} \backslash Q_{R 2}^{c}=\varnothing$.

- $q_{R}^{c} \in Q_{R 2}^{c} \backslash Q_{R 1}^{c}$. As we have $q_{R}^{c} \eta_{f} q_{A}^{c}$, condition 3 in $S R_{2}^{\tau} \sqsubseteq_{D_{2}} S A_{2}^{c}$ gives:

- either $q_{A}^{c} \nrightarrow$ and we are done,

- or $q_{A}^{c} \stackrel{e}{\rightarrow}_{A} q_{A}^{c \prime}$ and $q_{R}^{c} \in D_{2}$. As $q_{R}^{c} \notin Q_{R 1}^{c}$, we have a contradiction with $D_{2} \backslash Q_{R 1}^{c}=\varnothing$.

- $q_{R}^{c} \in Q_{R 1}^{c} \cap Q_{R 2}^{c}$. As we have $q_{R}^{c} \eta_{f} q_{A}^{c}$, condition 3 in $S R_{1}^{\tau} \sqsubseteq D_{1} S A_{1}^{c}$ gives:

- either $q_{A}^{c} \nrightarrow$ and we are done,

- or $q_{A}^{c} \stackrel{e}{\rightarrow}_{A} q_{A}^{c \prime}$ and $q_{R}^{c} \in D_{1}$.

Moreover, condition 3 in $S R_{2}^{\tau} \sqsubseteq D_{2} S A_{2}^{c}$ gives:

- either $q_{A}^{c} \nrightarrow$ and we are done,

- or $q_{A}^{c} \stackrel{e}{\rightarrow}_{A} q_{A}^{c \prime}, q_{R}^{c} \in D_{1}$ and $q_{R}^{c} \in D_{2}$. We have a contradiction with $D_{1} \cap D_{2}=\varnothing$. 


\section{Lemma 4 (Lack of $\tau$-divergence Condition)}

We must prove that there is no $\left(q_{R}^{c} \stackrel{\tau}{\rightarrow} q_{R}^{c \prime} \stackrel{\tau}{\rightarrow} q_{R}^{c} \prime \stackrel{\tau}{\rightarrow} \ldots \stackrel{\tau}{\rightarrow} \ldots\right)$ in $M$.

Proof by contradiction. We suppose that $\left(q_{R} \stackrel{\tau}{\rightarrow} q_{R}^{c} \stackrel{\tau}{\rightarrow} q_{R}^{c \prime \prime} \stackrel{\tau}{\rightarrow} \ldots \stackrel{\tau}{\rightarrow} \ldots\right)$ exists.

- $q_{R}^{c}, q_{R}^{c \prime}, q_{R}^{c \prime}, \ldots \in Q_{R 1}^{c}$. We have a contradiction with condition 4 for $S R_{1}^{c} \sqsubseteq S A_{1}^{c}$.

- $q_{R}^{c}, q_{R}^{c}, q_{R}^{c}{ }^{\prime \prime}, \ldots \in Q_{R 2}^{c}$. We have a contradiction with condition 4 for $S R_{2}^{c} \sqsubseteq S A_{2}^{c}$.

- $\exists i .\left(q_{R}^{c(i)} \in Q_{R 1}^{c} \wedge q_{R}^{c}{ }^{(i+1)} \in Q_{R 2}^{c}\right)$. Inconsistence with condition 3 for $S R_{1}^{\tau} \sqsubseteq S A_{1}^{c}$.

\section{Lemma 5 (Non-determinism Preservation Condition)}

Assume that $q_{A}^{c} \stackrel{\alpha}{\rightarrow} q_{A}^{c \prime}$; we must show that there exists $q_{R}^{c \prime}, q_{R}^{c \prime \prime}$ and $q_{A}^{c \prime \prime}$ such that $q_{R}^{c \prime} \eta q_{A}^{c}, q_{R}^{c \prime} \stackrel{\alpha}{\rightarrow} q_{R}^{c \prime \prime}, q_{A}^{c} \stackrel{\alpha}{\rightarrow} q_{A}^{c \prime \prime}$, and $q_{R}^{c \prime \prime} \eta q_{A}^{c \prime \prime}$.

Proof on the form of $\alpha$. There are three cases.

- $\alpha=\left(e_{1},-\right)$. By Definition 4, we have $\left(q_{A}^{c},\left(e_{1},-\right), q_{A}^{c \prime}\right) \in T_{A 1}^{c}, q_{A}^{c} \in Q_{A 1}^{c}$ and $q_{A}^{c \prime} \in Q_{A 1}^{c}$. Since $S R_{1}^{\tau} \sqsubseteq S A_{1}^{c}$, there exists $q_{R}^{c}$ such that $q_{R}^{c} \in Q_{R 1}^{c}$ and $q_{R}^{c} \eta q_{A}^{c}$. By condition 5 for $S R_{1}^{\tau} \sqsubseteq S A_{1}^{c}$, we obtain that there exist $q_{R}^{c \prime}, q_{R}^{c \prime \prime}$ and $q_{A}^{c \prime \prime}$ such that $q_{R}^{c \prime} \eta q_{A}^{c}, q_{R}^{c \prime} \stackrel{\alpha}{\rightarrow} q_{R}^{c \prime \prime}, q_{A}^{c} \stackrel{\alpha}{\rightarrow} q_{A}^{c \prime \prime}$ and $q_{R}^{c \prime \prime} \eta q_{A}^{c \prime \prime}$.

- $\alpha=\left(-, e_{2}\right)$. By Definition 4, we have $\left(q_{A}^{c},\left(-, e_{2}\right), q_{A}^{c \prime}\right) \in T_{A 2}^{c}, q_{A}^{c} \in Q_{A 2}^{c}$ and $q_{A}^{c \prime} \in Q_{A 2}^{c}$. Since $S R_{2}^{\tau} \sqsubseteq S A_{2}^{c}$, there exists $q_{R}^{c}$ such that $q_{R}^{c} \in Q_{R 2}^{c}$ and $q_{R}^{c} \eta q_{A}^{c}$. By condition 5 for $S R_{2}^{\tau} \sqsubseteq S A_{2}^{c}$, we obtain that there exist $q_{R}^{c \prime}, q_{R}^{c \prime \prime}$ and $q_{A}^{c \prime \prime}$ such that $q_{R}^{c \prime} \eta q_{A}^{c}, q_{R}^{c \prime} \stackrel{\alpha}{\rightarrow} q_{R}^{c \prime \prime}, q_{A}^{c} \stackrel{\alpha}{\rightarrow} q_{A}^{c \prime \prime}$ and $q_{R}^{c \prime \prime} \eta q_{A}^{c \prime \prime}$.

- $\alpha=\left(e_{1}, e_{2}\right)$. By Definition 4, we have $\left(q_{A}^{c},\left(e_{1}, e_{2}\right), q_{A}^{c \prime}\right) \in T_{A 1}^{c}, q_{A}^{c} \in Q_{A 1}^{c}$ and $q_{A}^{c \prime} \in Q_{A 2}^{c}$. Since $S R_{1}^{\tau} \sqsubseteq S A_{1}^{c}$, there exists $q_{R}^{c}$ such that $q_{R}^{c} \in Q_{R 1}^{c}$ and $q_{R}^{c} \eta q_{A}^{c}$. By condition 5 for $S R_{1}^{\tau} \sqsubseteq S A_{1}^{c}$, we obtain that there exist $q_{R}^{c \prime}, q_{R}^{c \prime \prime}$ and $q_{A}^{c \prime \prime}$ such that $q_{R}^{c \prime} \eta q_{A}^{c}, q_{R}^{c \prime} \stackrel{\alpha}{\rightarrow} q_{R}^{c \prime \prime}, q_{A}^{c} \stackrel{\alpha}{\rightarrow} q_{A}^{c \prime \prime}$ and $q_{R}^{c \prime \prime} \eta q_{A}^{c \prime \prime}$.

$\mathrm{RR} \mathrm{n}^{\circ} 4862$ 


\section{A.2 $[R] \Rightarrow[a] \wedge[b] \wedge[c]$}

Property 1 gives immediately $D_{12}=\varnothing[c]$ and $S R_{1}\left\|_{S y n c h^{\prime}} S R_{2} \sqsubseteq_{D_{12}} S A_{1}\right\|_{S y n c h} S A_{2}$ $\left[R^{\prime}\right]$. We show that $\left[R^{\prime}\right]$ implies $[a]$ and $[b]$.

Let $M_{1} \stackrel{\text { def }}{=}\left\{\left(q_{R 1}^{c}, q_{A 1}^{c}\right) \mid\left(q_{R 1}^{c} \in Q_{R 1}^{c} \wedge q_{A 1}^{c} \in Q_{A 1}^{c}\right\}\right.$

and $M_{2} \stackrel{\text { def }}{=}\left\{\left(q_{R 2}^{c}, q_{A 2}^{c}\right) \mid\left(q_{R 2}^{c} \in Q_{R 2}^{c} \wedge q_{A 2}^{c} \in Q_{A 2}^{c}\right\}\right.$ be two sets

with $\forall q_{R}^{c} \cdot\left(q_{R}^{c} \in Q_{R 1}^{c} \cup Q_{R 2}^{c} \Rightarrow \exists q_{A}^{c} \cdot\left(q_{A}^{c} \in Q_{A 1}^{c} \cup Q_{A 2}^{c} \wedge q_{R}^{c} \eta_{f} q_{A}^{c}\right)\right)$.

We show that $M_{1}$ verifies the conditions of Definition 8 using Lemmas 6, 7, 8, 9 and 10. The proof is the same for $M_{2}$.

As $D_{12}=\varnothing$, we always have $\left[c^{\prime}\right]:\left(D_{1} \cap D_{2}=\varnothing\right) \wedge\left(D_{1} \backslash Q_{R 2}^{c}=\varnothing\right) \wedge\left(D_{2} \backslash Q_{R 1}^{c}=\varnothing\right)$.

\section{Lemma 6 (Strict Transition Refinement Condition)}

Assume that $q_{R 1}^{c} \stackrel{\alpha}{\rightarrow} q_{R 1}^{c}$; we must prove that there exists $q_{A 1}^{c}{ }^{\prime}$ such that $q_{A 1}^{c} \stackrel{\alpha}{\rightarrow} q_{A 1}^{c}{ }^{\prime}$ and $q_{R}^{c \prime} \eta q_{A}^{c}{ }^{\prime}$.

Proof on the form of $\alpha$. There are three cases.

- $\alpha=\left(e_{1},-\right)$ or $\alpha=\left(e_{1}, e_{2}\right)$. By Definition 4, we have $q_{R 1}^{c} \in Q_{R 1}^{c}$ and $q_{R 1}^{c}{ }^{\prime} \in Q_{R 1}^{c}$. Since $q_{R 1}^{c} \eta_{f} q_{A 1}^{c}$ for the whole system, we obtain that there exist $q_{A 1}^{c}$ and $q_{A 1}^{c}$ 'such that $q_{A 1}^{c}, q_{A 1}^{c}{ }^{\prime} \in Q_{A}^{c}, q_{A 1}^{c} \stackrel{\alpha}{\rightarrow} q_{A 1}^{c}{ }^{\prime}$ and $q_{R}^{c}{ }^{\prime} \eta q_{A}^{c}{ }^{\prime}$. By Definition 4, we have $q_{A 1}^{c}$ and $q_{A 1}^{c}{ }^{\prime}$ belonging to $Q_{A 1}^{c}$. We conclude that $\left(q_{R 1}^{c}, q_{A 1}^{c}\right) \in M_{1}$.

- $\alpha=\left(-, e_{2}\right)$. Impossible because of Definition 4 .

\section{Lemma 7 (Stuttering Transition Refinement Condition)}

Assume $q_{R 1}^{c} \stackrel{\tau}{\rightarrow} q_{R 1}^{c}{ }^{\prime}$; we must prove that $q_{R 1}^{c}{ }^{\prime} \eta q_{A 1}^{c}$.

Proof on the form of labels covered by $\tau$. There are three cases.

- $\left(e_{1}^{\prime},-\right) \backslash \tau$ or $\left(e_{1}^{\prime}, e_{2}^{\prime}\right) \backslash \tau$. By Definition 4, we have $q_{R 1}^{c} \in Q_{R 1}^{c}$ and $q_{R 1}^{c}{ }^{\prime} \in Q_{R 1}^{c}$. Since $q_{R 1}^{c} \eta_{f} q_{A 1}^{c}$ for the whole system, we obtain that there exists $q_{A 1}^{c}$ such that $q_{A 1}^{c} \in Q_{A}^{c}$ and $q_{R 1}^{c}{ }^{\prime} \eta q_{A 1}^{c}$.

- If $q_{A 1}^{c} \in Q_{A 1}^{c}$, we conclude that $\left(q_{R 1}^{c}, q_{A 1}^{c}\right) \in M_{1}$.

- If $q_{A 1}^{c} \in Q_{A 2}^{c} \backslash Q_{A 1}^{c}$, then $\left(q_{R 1}^{c}, q_{A 1}^{c}\right) \notin M_{1}$ and we are done.

- $\left(-, e_{2}^{\prime}\right) \backslash \tau$. Impossible because of Definition 4. 


\section{Lemma 8 (Old or New Deadlocks Condition)}

Assume that $q_{R 1}^{c} \nrightarrow$ with $q_{R 1}^{c} \in Q_{R 1}^{c}$; we must prove that either $q_{A 1}^{c} \nrightarrow$ or $\exists q_{A 1}^{c}{ }^{\prime} \cdot\left(q_{A 1}^{c} \stackrel{e}{\rightarrow}\right.$ $q_{A 1}^{c}{ }^{\prime}$ and $\left.q_{R 1}^{c} \in D_{1}\right)$.

- Suppose that $q_{R 1}^{c}$ is a deadlock for $S R_{1} \|_{S y n c h^{\prime}} S R_{2}$. As we have $q_{R 1}^{c} \eta_{f} q_{A 1}^{c}$ for the whole system, we have two cases:

- either $q_{A 1}^{c} \nrightarrow$;

- If $q_{A 1}^{c} \in Q_{A 1}^{c}$, we conclude that $\left(q_{R 1}^{c}, q_{A 1}^{c}\right) \in M_{1}$.

- If $q_{A 1}^{c} \in Q_{A 2}^{c} \backslash Q_{A 1}^{c}$, then $\left(q_{R 1}^{c}, q_{A 1}^{c}\right) \notin M_{1}$ and we are done.

- or $\exists q_{A 1}^{c}{ }^{\prime} \cdot\left(q_{A 1}^{c} \stackrel{e}{\rightarrow} q_{A 1}^{c}{ }^{\prime} \wedge q_{R 1}^{c} \in D_{12}\right)$. Impossible since $D_{12}=\varnothing$.

- Suppose that $q_{R 1}^{c}$ is not a deadlock for $S R_{1} \|_{S y n c h^{\prime}} S R_{2}$. Since $q_{R 1}^{c} \nrightarrow$ for $S R_{1}$, then $q_{R 1}^{c} \stackrel{e}{\rightarrow} q_{R 1}^{c}{ }^{\prime}$ for $S R_{2}$. Then we have $q_{R 1}^{c} \in Q_{R 2}^{c}$. We have $q_{R 1}^{c} \eta_{f} q_{A 1}^{c}$ for the whole system and:

- either $q_{A 1}^{c} \nrightarrow$;

- If $q_{A 1}^{c} \in Q_{A 1}^{c}$ we conclude that $\left(q_{R 1}^{c}, q_{A 1}^{c}\right) \in M_{1}$.

- If $q_{A 1}^{c} \in Q_{A 2}^{c} \backslash Q_{A 1}^{c}$ then $\left(q_{R 1}^{c}, q_{A 1}^{c}\right) \notin M_{1}$ and we are done.

- or $\exists q_{A 1}^{c}{ }^{\prime} \cdot\left(q_{A 1}^{c} \stackrel{e}{\rightarrow} q_{A 1}^{c}{ }^{\prime}\right)$;

- If $q_{A 1}^{c} \in Q_{A 1}^{c}$, we verify $D_{1} \backslash Q_{R 2}^{c}=\varnothing$ because of $q_{R 1}^{c} \in Q_{R 2}^{c}$ and we conclude that $\left(q_{R 1}^{c}, q_{A 1}^{c}\right) \in M_{1}$.

- If $q_{A 1}^{c} \in Q_{A 2}^{c} \backslash Q_{A 1}^{c}$, then $\left(q_{R 1}^{c}, q_{A 1}^{c}\right) \notin M_{1}$ and we are done.

\section{Lemma 9 (Lack of $\tau$-divergence Condition)}

We must prove that there is no $\left(q_{R 1}^{c} \stackrel{\tau}{\rightarrow} q_{R 1}^{c}{ }^{\prime} \stackrel{\tau}{\rightarrow} q_{R 1}^{c}{ }^{\prime \prime} \stackrel{\tau}{\rightarrow} \ldots \stackrel{\tau}{\rightarrow} \ldots\right)$ in $M_{1}$.

Proof by contradiction. We suppose that $\left(q_{R 1}^{c} \stackrel{\tau}{\rightarrow} q_{R 1}^{c}{ }^{\prime} \stackrel{\tau}{\rightarrow} q_{R 1}^{c}{ }^{\prime \prime} \stackrel{\tau}{\rightarrow} \ldots \stackrel{\tau}{\rightarrow} \ldots\right)$ exists.

Since $q_{R 1}^{c}, q_{R 1}^{c}{ }^{\prime}, q_{R 1}^{c}{ }^{\prime \prime}, \ldots \in Q_{R}^{c}$, we have a $\tau$-divergence for the whole system.

\section{Lemma 10 (Non-determinism Preservation Condition)}

Assume that $q_{A 1}^{c} \stackrel{\alpha}{\rightarrow} q_{A 1}^{c}{ }^{\prime}$; we must show that there exist $q_{R 1}^{c}{ }^{\prime}, q_{R 1}^{c}$ " and $q_{A 1}^{c}{ }^{\prime \prime}$ such that $q_{R 1}^{c}{ }^{\prime} \eta q_{A 1}^{c}, q_{R 1}^{c}{ }^{\prime} \stackrel{\alpha}{\rightarrow} q_{R 1}^{c}{ }^{\prime \prime}, q_{A 1}^{c} \stackrel{\alpha}{\rightarrow} q_{A 1}^{c}{ }^{\prime \prime}$, and $q_{R 1}^{c}{ }^{\prime \prime} \eta q_{A 1}^{c}{ }^{\prime \prime}$.

Proof on the form of $\alpha$. There are three cases.

- $\alpha=\left(e_{1},-\right)$ or $\alpha=\left(e_{1}, e_{2}\right)$. By Definition 4, we have $q_{A 1}^{c} \in Q_{A 1}^{c}$ and $q_{A 1}^{c}{ }^{\prime} \in Q_{A 1}^{c}$. Since $q_{R 1}^{c} \eta_{f} q_{A 1}^{c}$ for the whole system, we obtain that there exist $q_{R 1}^{c}{ }^{\prime}$, $q_{R 1}^{c}$ " and $q_{A 1}^{c}$ " such that $q_{R 1}^{c}{ }^{\prime} \eta q_{A 1}^{c}, q_{R 1}^{c}{ }^{\prime} \stackrel{\alpha}{\rightarrow} q_{R 1}^{c}{ }^{\prime \prime}, q_{A 1}^{c} \stackrel{\alpha}{\rightarrow} q_{A 1}^{c}$ " and $q_{R 1}^{c}{ }^{\prime \prime} \eta q_{A 1}^{c}$ ". By Definition 4, we conclude that $q_{R 1}^{c}{ }^{\prime}$ and $q_{R 1}^{c}$ " belong to $Q_{R 1}^{c}$, and that $q_{A 1}^{c}$ " belongs to $Q_{A 1}^{c}$. We conclude that $\left(q_{R 1}^{c}, q_{A 1}^{c}\right) \in M_{1}$.

- $\alpha=\left(-, e_{2}\right)$. Impossible because of Definition 4. 


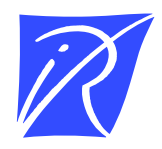

Unité de recherche INRIA Lorraine

LORIA, Technopôle de Nancy-Brabois - Campus scientifique 615, rue du Jardin Botanique - BP 101 - 54602 Villers-lès-Nancy Cedex (France)

Unité de recherche INRIA Rennes : IRISA, Campus universitaire de Beaulieu - 35042 Rennes Cedex (France) Unité de recherche INRIA Rhône-Alpes : 655, avenue de l'Europe - 38330 Montbonnot-St-Martin (France) Unité de recherche INRIA Rocquencourt : Domaine de Voluceau - Rocquencourt - BP 105 - 78153 Le Chesnay Cedex (France) Unité de recherche INRIA Sophia Antipolis : 2004, route des Lucioles - BP 93 - 06902 Sophia Antipolis Cedex (France) 